\title{
Shigeto Kawahara* and Gakuji Kumagai What voiced obstruents symbolically represent in Japanese: evidence from the Pokémon universe
}

\author{
https://doi.org/10.1515/jjl-2021-2031
}

\begin{abstract}
Kawahara, Noto, and Kumagai (2018b) found that within the corpus of existing Pokémon names, the number of voiced obstruents in the characters' names correlates positively with their weight, height, evolution levels and attack values. While later experimental studies to some extent confirmed the productivity of these sound symbolic relationships (e.g. Kawahara and Kumagai 2019a), they are limited, due to the fact that the visual images presented to the participants primarily differed with regard to evolution levels. The current experiments thus for the first time directly explored how each of these semantic dimensions-weight, height, evolution levels, and attack values-correlates with the number of voiced obstruents in nonce names. The results of two judgment experiments show that all of these parameters indeed correlate positively with the number of voiced obstruents in the names. Overall, the results show that a particular class of sounds-in our case, a set of voiced obstruentscan signal different semantic meanings within a single language, supporting the pluripotentiality of sound symbolism (Winter, Pérez-Sobrino, and Brown 2019). We also address another general issue that has been under-explored in the literature on sound symbolism; namely, its cumulative nature. In both of the experiments, we observe that two voiced obstruents evoke stronger images than one voiced obstruent, instantiating what is known as the counting cumulativity effect (Jäger and Rosenbach 2006).
\end{abstract}

Keywords: cumulativity; Japanese; pluripotentiality; Pokémon names; soundsymbolism; voiced obstruents

\section{Introduction}

In recent years, we have witnessed a rapidly increasing body of studies on sound symbolism-systematic iconic associations that hold between sounds and

*Corresponding author: Shigeto Kawahara, Keio University, Tokyo, Japan, E-mail: kawahara@icl.keio.ac.jp

Gakuji Kumagai, Meikai University, Urayasu, Japan, E-mail: gakujik@meikai.ac.jp 
meanings (e.g. Dingemanse et al. 2015; Kawahara 2020b; Lockwood and Dingemanse 2015; Nuckolls 1999; Sidhu and Pexman 2018, 2019 for recent reviews). Contrary to the received wisdom in modern linguistic thinking in the twentieth century that the relationships between sounds and meanings are in principle arbitrary (Hockett 1959; Saussure 1916), phonetic and psychological research consistently shows that some systematic iconic relationships hold between these two dimensions, which can arguably be construed as instances of more general cross-modal associations, commonly observed in human cognition patterns (Spence 2011).

Within this research paradigm, Kawahara et al. (2018b) studied the corpus of existing Pokémon names in Japanese. Pokémon is a video game series which was first developed by Nintendo Inc. in 1996, and has become very popular worldwide since then. In this game series, players collect fictional creatures called Pokémon (truncation of [poketto monsutaa] 'pocket monster'), train them, and have them fight with other Pokémon creatures. In this game series, some Pokémon characters evolve into different characters (e.g. Pikachu becomes Raichu), and when they do so, they generally become larger, heavier, and stronger. When Kawahara et al. (2018b) analyzed the corpus of these Pokémon names in 2017, there were more than 700 characters. Shih et al. (2019) reported a replication of this study with an extended dataset $(N=802)$, including those characters which came out after Kawahara et al.'s (2018) analysis. This new study also targets the Pokémon names from a wide range of languages, including Cantonese, English, Japanese, Korean, Mandarin and Russian; their findings show intriguing cross-linguistic similarities and differences among the sound symbolic patterns in these target languages.

The Pokémon corpus offers a nice testing ground for cross-linguistic studies of sound symbolism for several reasons. One is that since each Pokémon character is specified for its weight, height, evolution levels, and strength parameters, it allows us to conduct quantitative analyses of possible sound symbolic patterns in real names. Second, the size of the corpus is much larger than what is usually analyzed in the studies of sound symbolism in real words (e.g. 40 basic vocabulary items: Blasi et al. 2016 and Wichmann et al. 2010; 28 antonym pairs: Johansson and Zlatev 2013; 112 male names and 151 female names: Pitcher et al. 2013). ${ }^{1}$ The third virtue of using the Pokémon universe to study sound symbolic patterns is that, since the Pokémon universe is fixed across different languages, it allows for a systematic cross-linguistic comparison of sound symbolic patterns (Shih et al. 2018, 2019)-this is not trivial, as

\footnotetext{
1 Some of these studies analyze basic vocabulary items in many languages, and we thus do not intend to claim that studying the Pokémon lexicon is inherently superior to these other studies. These different studies have different advantages, and aim to address different questions, although ultimately, we are all interested in explicating the nature of sound symbolism in natural languages.
} 
languages differ in the set of real-world attributes that they refer to. For example, while Japanese lexically distinguishes "younger sisters" (imooto) from "older sisters" (ane), English does not make this lexical distinction. This sort of cross-linguistic difference makes the cross-linguistic comparison of sound symbolism difficult; on the other hand, the Pokémon lexicon is fixed across all the languages and thus avoids this problem (Shih et al. 2018, 2019). ${ }^{2}$

With these research advantages in mind, Kawahara et al. (2018b) found, for example, that the number of voiced obstruents (e.g. [b], [d], [g], [z]) in the Japanese Pokémon character names generally correlates positively with their weight, height, evolution levels, and attack values. One of the questions that arose from this study is the productivity of these sound symbolic relationships, the primary research question being whether the sound symbolic patterns that exist in the Pokémon corpus hold among general Japanese speakers, or whether they are instead limited to the small group of the Pokémon designers. Several studies therefore performed a forced-choice task by presenting a pair of new pre-evolution and post-evolution Pokémon characters, sometimes the post-evolution characters being about 1.5 times as large as the pre-evolution characters (Kawahara et al. 2018a; Kawahara and Kumagai, 2019a; Kumagai and Kawahara 2019). The general findings of these studies were that native speakers of Japanese, both children and adults, tend to associate nonce names having voiced obstruents with larger, postevolution characters.

While these studies offer some evidence for the productivity of sound symbolic associations in the Pokémon names, what remains unclear is which semantic dimension was symbolically signaled by voiced obstruents in these experiments-evolution level or height, or both? It could be the case, for example, that voiced obstruents were sound-symbolically associated with evolution levels only, but since in the Pokémon world, more evolved characters were generally larger, we may have observed the effects of voiced obstruents on height, but only indirectly. If that was the case, we should not conclude that voiced obstruents symbolically represent large size in Japanese, independent of evolution levels. Another aspect in which these previous experimental studies on Pokémon names were limited is that they did not directly test the sound symbolic

2 This is true only to the extent that Pokémon is available in that language. However, even if Pokémon names are not translated into a particular language, we can run an elicitation study to examine how Pokémon creatures would be named in that language, thereby creating a new corpus of Pokémon names. For an example of this sort of study targeting Brazilian Portuguese, see Godoy et al. (2020). Kawahara and Kumagai (2019a), which Godoy et al. (2020) heavily built upon, also report an elicitation study targeting Japanese speakers. 
effects of voiced obstruents on weight or attack values at all. ${ }^{3}$ To overcome these limitations, the current study set out to address precisely which semantic dimension is associated with voiced obstruents in Japanese.

Previous studies on the sound symbolic values of voiced obstruents in Japanese, outside the context of the Pokémon-related research, point out some specific images of voiced obstruents, such as dirtiness (Kawahara et al. 2008; Kawahara 2017), general negative images (Kubozono 1999; Suzuki 1962) and largeness (Shinohara and Kawahara 2016). Hamano (1998) suggests that in the Japanese ideophone system (a.k.a. mimetics), voiced obstruents can mean "heavy," "large," "coarse" and "thick" (p. 83). However, many of such studies are based on impressionistic observations (except for Kawahara et al. 2008; Shinohara and Kawahara 2016), and no systematic experimental studies have been conducted to address what semantic dimension can be signaled by voiced obstruents in Japanese. In this sense, the current study expands on these previous observations and aims to advance our understanding of what voiced obstruents can symbolically represent in Japanese.

However, the current research goes well beyond mere specific case studies of sound symbolic patterns in Japanese, offering insights that bear on some current issues in sound symbolism research. First, one prominent theory of sound symbolism is the Frequency Code Hypothesis (Ohala 1983, 1994), which suggests that sounds with high frequency energy denote something small, because the size of a resonance chamber negatively correlates with its resonance frequency. Thus, the Frequency Code Hypothesis alone predicts that it is size (but nothing else) that can be signaled sound symbolically via manipulation of frequency. Since voiced obstruents involve low frequency energy (e.g. Chodroff and Wilson 2014; Kingston and Diehl 1994, 1995; Stevens and Blumstein 1981), it makes sense from this point of view that voiced obstruents are associated with image of largeness. However, the Frequency Code Hypothesis alone does not predict anything about the relationship between frequency and weight, for example. One should note, however, that Ohala $(1983,1994)$ himself goes beyond the matter of size alone-he argues, for instance, that we generally smile with an "[i]-face” because [i] has high second formant frequency energy, which signals smallness, and is thus suited to express a lack of hostility. Gussenhoven (2004) suggests that many languages use a rising intonation (i.e. a H-tone) for interrogative sentences, because a $\mathrm{H}$-tone is, by virtue

3 In a related study, by way of a corpus study and a judgment experiment, Kawahara et al. (2020) studied the effects of voiced obstruents on attack values using Pokémon's move ("waza") names, which they use during their battles. In the judgment experiment, they found a positive effect of voiced obstruents on judged attack values, although they did not manipulate the number of voiced obstruents in their stimuli, unlike the current experiments reported below. The effect of voiced obstruents on attack values in the existing move names was tangible in the simple regression analysis, but was not significant under the multiple regression analysis. 
of sounding "small," suited to express "submissiveness," a property that may be desirable when one is looking for information (see also Nuckolls 1999; Gussenhoven 2016).

It may be the case, then, that the Frequency Code associates resonance frequency with size, but size can be semantically associated with other concepts, such as weight, lack of animosity, or submissiveness. This nature of sound symbolism - that a certain set of sounds can be associated with multiple meanings-has recently been referred to as pluripotentiality of sound symbolism (Winter et al. 2019), and we are only starting to understand to what extent pluripotentiality holds in sound symbolic patterns in human languages. In short, despite the accumulation of case studies on sound symbolism (Dingemanse et al. 2015; Kawahara 2020b; Lockwood and Dingemanse 2015; Nuckolls 1999; Sidhu and Pexman 2018, 2019), exploration of what kinds of sounds can symbolically represent what in what language remains an important yet still under-explored question in the sound symbolism research. By way of a case study, this paper attempts to address this general issue, using the Pokémon universe as the testing ground. To this end, we report two experiments that examine the question of which semantic properties voiced obstruents can symbolically represent in Japanese.

In addition to addressing the question of precisely what voiced obstruents can symbolically represent in Japanese, the current experiments aim to address one general issue that has been understudied in the literature on sound symbolism; namely, the question of cumulativity. In the current context, this issue boils down to the question of whether sound symbolic effects of multiple occurrences of the same class of segments add up or not. This question of cumulativity is important to address for two reasons. The first reason is empirical: we do not have sufficient data to know about cumulative effects in sound symbolism. There are a few impressionistic reports which suggest that sound symbolic effects are cumulative. For example, Hamano (2013) reports that in the ideophone system of the Tsugaru dialect of Japanese, two voiced obstruents evoke stronger iconic meanings than one voiced obstruent. Likewise, Martin (1962), cited by McCarthy (1983), points out that in Korean ideophones, tense consonants function as intensifiers, and the degree of intensification is stronger when there are two segments than when there is one. Thompson and Estes (2011) built upon the observations that some sounds are associated with images of largeness (e.g. Sapir 1929), and found by way of experimentation that the larger the object, the more likely it is that the assigned names contain such "large phonemes." See Kawahara (2020a) for a recent summary for other possible cases of cumulative effects in sound symbolism.

For a theoretical reason also, studying cumulative effects in sound symbolism is important. Indeed, cumulativity is one issue that is actively debated in current linguistic theorization, especially in the context of comparing Optimality Theory (Prince and Smolensky 1993/2004) and other related constraint-based theories, 
such as Harmonic Grammar (Legendre et al. 1990; Pater 2009). Optimality Theory, which has been the dominant framework for phonological analyses since the early 90s, posits that constraints are ranked, whereas Harmonic Grammar posits that constraints are weighted. The latter theory predicts cumulative behaviors of constraint violations, whereas in Optimality Theory, this is not necessarily the case. More specifically, in Optimality Theory, when Constraint A is ranked above Constraint $B$, then a single violation of Constraint $A$ is considered to be more important than any number of violations of Constraint B-violations of Constraint $B$ are not cumulative. In Harmonic Grammar, on the other hand, even when Constraint A has a higher weight than Constraint $\mathrm{B}$, multiple violations of Constraint B can be considered to be more severe than a single violation of Constraint A. See Kawahara (2020a), Kawahara (2020c), Pater (2009), Prince and Smolensky (1993/2004) and Jäger and Rosenbach (2006) for detailed explications of this difference.

At a more general level, whether linguistic patterns show cumulative effects or not is an important issue to address in current linguistic theorization, because it crucially bears on the question of what kind of mechanism should be used to model our linguistic knowledge. To the extent that sound symbolism is a part of the core linguistic system (Alderete and Kochetov 2017; Kawahara et al. 2019; Kawahara 2020b), exploring whether sound symbolism shows a cumulative pattern or not can shed light on this on-going debate (Kawahara 2020c). If cumulativity is the norm, the mechanism that lies behind our linguistic knowledge should be the one that naturally predicts cumulative effects, such as Maximum Entropy Harmonic Grammar (e.g. Breiss 2020; Breiss and Albright 2020; Goldwater and Johnson 2003; Hayes and Wilson 2008; Hayes et al. 2012; Kawahara 2020a, 2020c; McPherson and Hayes 2016).

To address these two questions-pluripotentiality and cumulativity-we report two experiments which tested whether voiced obstruents in Japanese are symbolically associated with size, weight, strength and evolution levels; in these experiments, we varied the number of voiced obstruents from zero to two, in order to examine whether we observe cumulative effects of voiced obstruents in these sound symbolic patterns.

\section{Experiment 1}

The first experiment explored whether weight and strength (=attack values) can be signaled by voiced obstruents in the names. We did not test all four semantic dimensions that are of interest (weight, height, evolution status, and strength) in a single experiment, because we expected that that would put too much burden on 
our participants; we hence instead ran two separate experiments, each testing two semantic dimensions. Since evolution levels and attack values are arguably semantically more complex concepts than weight and height, they are separately tested in different experiments.

The whole experiment was blocked into two sections. The first section tested the effects of voiced obstruents on the judgment of weight; the second section tested their effects on the judgment of attack values.

\subsection{Methods}

\subsubsection{Procedure}

In this experiment, the participants were told that the experiment was about making judgments about new Pokémon names. They were told at the beginning of the experiment that the experiment consists of two parts. In the first part, they were provided with one nonce Pokémon name per trial, and asked to judge its weight, using a range from 1 to $100 \mathrm{~kg}$. They provided their response by adjusting a slider. In the second part, they were also provided with one nonce Pokémon name, and were asked to judge its attack value, again using a slider ranging from 1 to 100 (no units were provided). The order of the questions within a block was randomized per participant. All of the stimulus names were presented in the katakana orthography, which is used for nonce words as well as for real Pokémon names. The experiment was conducted online using SurveyMonkey. Unlike the previous Pokémonastics experiments (Kawahara and Kumagai 2019a; Kumagai and Kawahara 2019), no pictures of Pokémon were presented, since those pictures that were used in these experiments differ in terms of size, evolution levels, and more generally, how they look.

\subsubsection{Stimuli}

All the stimuli were trisyllabic, consisting of light CV syllables only. The main manipulation was the number of voiced obstruents $(=[\mathrm{b}],[\mathrm{d}],[\mathrm{g}],[\mathrm{z}],[\mathrm{z}],[\mathrm{dz}])$ contained in the names, consisting of three conditions: no voiced obstruents, one voiced obstruent, and two voiced obstruents. Ten nonce items were prepared for each condition, as shown in Table 1 . All the names were created using an online nonce-word generator, in order to avoid the experimenters' bias to pre-select stimuli that were likely to work (Westbury 2005). This name generator randomly 
Table 1: The list of stimuli used in the current experiments. $[z]=$ a voiced alveopalatal fricative; $[\mathrm{dz}]=$ a voiced alveo-palatal affricate.

\begin{tabular}{lll}
\hline No voi obs & $\mathbf{1}$ voi obs & $\mathbf{2}$ voi obs \\
\hline [ku6iju] & [bitahe] & [gebiki] \\
[suфuma] & [birejo] & [zadoja] \\
[juфui] & [ganija] & [gibuse] \\
[neriru] & [bejumi] & [zugawa] \\
[6ihone] & [bojat6i] & [zadani] \\
[karutsu] & [bikohe] & [baboçi] \\
[jakama] & [baheho] & [dadera] \\
[sawake] & [geseci] & [zozike] \\
[rihojo] & [dzihana] & [zedot6i] \\
[sojuki] & [bijuri] & [dziboru] \\
\hline
\end{tabular}

combines Japanese (C)V-moras to create novel names. ${ }^{4}$ In the one voiced obstruent condition, the voiced obstruents occurred name-initially, as word-initial positions have well-known psycholinguistic salience (e.g. Browman 1978; Hawkins and Cutler 1988; Nooteboom 1981), and are known to evoke stronger sound symbolic images than word-internal segments (Kawahara et al. 2008). In the two voiced obstruent condition, they were placed in the first and second syllables. The same stimuli were used in the first and second blocks.

\subsubsection{Participants}

The call-for-participant of this experiment was circulated on various SNS (Social Networking Service) sites. Seventy native speakers of Japanese completed the survey. However, five of them were eliminated from the subsequent analysis, because they reported they had studied sound symbolism before. The data from the remaining sixty-five participants entered into the following analysis. Twentyone of them reported that they are male speakers. Most of them (forty-nine participants) were university students, and hence younger than 30 years old. No participants reported that they had participated in a similar Pokémon related experiment before.

4 This generator can be accessed at the following URL: http://bit.ly/2iGaKko (last access, August 2020). 


\subsubsection{Statistical analyses}

Since different speakers used a different range for their responses, the responses were standardized within each speaker. For statistical assessment, a linear mixed effects model was fit with standardized judged values as the dependent variable and the number of voiced obstruents as the fixed independent variable. The analyses were implemented using R ( $R$ Development Core Team 1993) and the lme4 package (Bates et al. 2017). Both speakers and items were included as random variables. A model with both random slopes and intercepts was fit first (Barr 2013; Barr et al. 2013); however, since the model with maximum random structure failed to converge in several cases, we interpreted the simpler model with random intercepts only in this paper. The $p$-values for the fixed effect were computed using the lmerTest package (Kuznetsova et al. 2017). The reference level for the independent variable was set to be " 1 voiced obstruent," which made it possible to compare the two pairs of the two adjacent conditions (i.e. 0 vs. 1 and 1 vs. 2 ).

\subsection{Results and discussion}

Figure 1 is a boxplot which shows the distribution of average judged weight values by participant for each condition; the grand averages in each condition are shown as white circles $(0=-0.59 ; 1=0.00 ; 2=0.58)$; the error bars around the

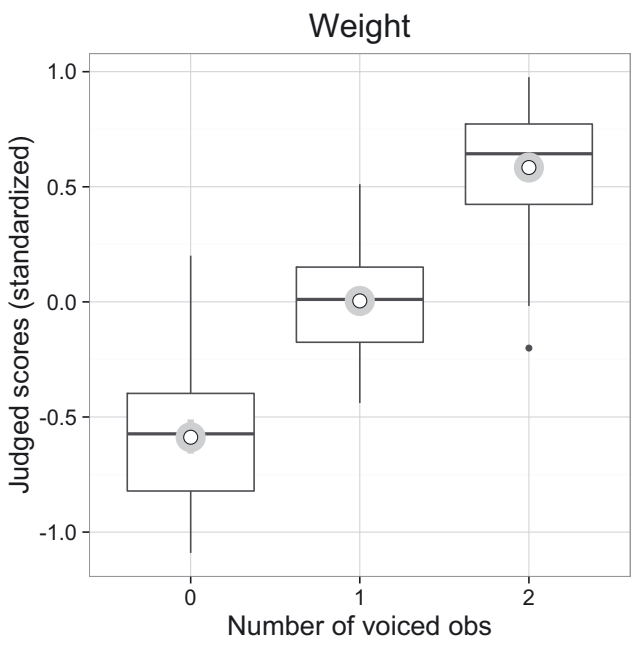

Figure 1: The boxplot representation of the by-participant distributions of the averaged judged weight values for each condition (standardized). The white circles represent the grand averages. The error bars around the averages represent the bootstrap 95\% confidence intervals. 
averages represent the bootstrap 95\% confidence intervals. We observe that the more voiced obstruents the names contain, the heavier they were judged to be. The differences between the two adjacent levels were both significant ( 0 vs. 1: $\beta=-12.25$, s.e. $=2.48, t=-4.9, p<.001 ; 1$ vs. $2: \beta=11.57$, s.e. $=2.48, t=4.66, p<.001)$. The current result for the first time provides unambiguous experimental support for the productivity of the weight-related sound symbolic association in Japanese, which was shown to hold in the existing Pokémon names (Kawahara et al. 2018b).

Figure 2 shows the results of judged attack values. Like the results on weight in Figure 1, the judged attack values show steady increase, as the number of voiced obstruents in the name increases (grand averages: $0=-0.44 ; 1=-0.08 ; 2=0.53$ ). The differences between the two adjacent conditions were both significant ( 0 vs. 1 : $\beta=-7.22$, s.e. $=2.12, t=-3.40, p<.01 ; 1$ vs. $2: \beta=12.44$, s.e. $=2.12, t=5.85, p<.001$ ). This result is compatible with the pattern observed in the existing Pokémon names (Kawahara et al. 2018b). It also expands on the observation by Kawahara et al. (2020), who show that Pokémon move names with a voiced obstruent are judged to be stronger than those names without voiced obstruents. Outside of the Pokémon related research, furthermore, this result accords well with the study by Sell et al. (2010), which found that a lower pitched voice is generally associated with strength.

Overall, Experiment 1 shows that Japanese speakers associate voiced obstruents with both weight and attack values. Moreover, the sound symbolic effects of voiced obstruents instantiate clear cases of a counting cumulativity effect (Jäger and

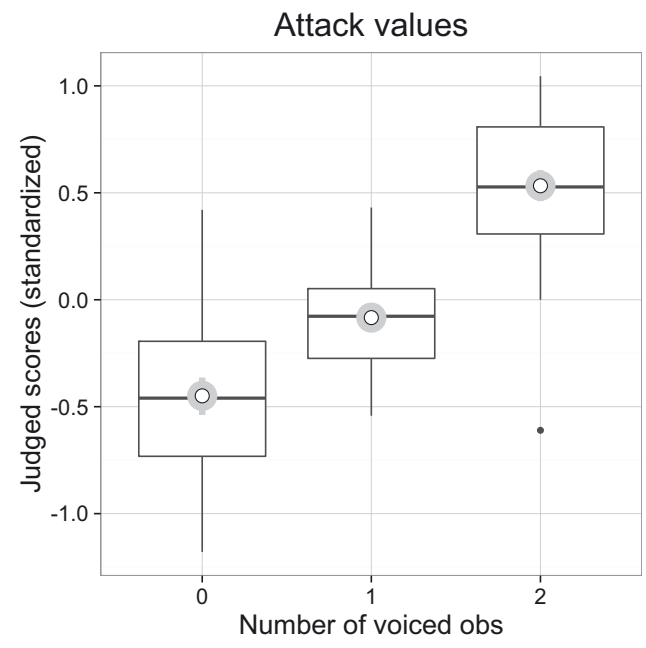

Figure 2: The boxplot representation of the average judged attack values. 
Rosenbach 2006 $)^{5}$-it is not only the presence of voiced obstruents, but also the number of voiced obstruents in the name that matters. This is not trivial, since whether the sound symbolic effects of different segments of the same kind can add up or not in a sound symbolic pattern is understudied (modulo Hamano 2013; Martin 1962; Thompson and Estes 2011). To the extent that sound symbolic connections constitute a part of core grammatical knowledge, similar to phonological input-output mapping (Alderete and Kochetov 2017; Kawahara et al. 2019; Kawahara 2020b, 2020c), it supports the idea that grammatical knowledge should be modeled using a mechanism which naturally predicts such cumulativity effects, of which Maximum Entropy Harmonic Grammar is a typical example (e.g. Breiss 2020; Goldwater and Johnson 2003; Hayes et al. 2012; Hayes and Wilson 2008; Kawahara 2020c; McPherson and Hayes 2016). See Kawahara (2020a) who develops analyses of various cases of cumulative effects in sound symbolism using this Maximum Entropy Grammar framework.

\section{Experiment 2}

\subsection{Methods}

Experiment 2 tested the effects of voiced obstruents on height (or size) and evolution levels. The procedure for Experiment 2 was almost identical to that of Experiment 1, except that when the participants made judgments regarding evolution levels, they were asked to make a binary choice (pre-evolution or postevolution) rather than using a scale, and hence accordingly, a logistic mixed effects model was used for the statistical analysis (Jaeger 2008). The questions regarding height were presented in the first block, and the questions regarding evolution levels were presented in the second block. The scale that was used for the height judgment ranged from 0 to $5 \mathrm{~m}$.

Fifty-eight native speakers of Japanese completed Experiment 2, but twelve of them had to be excluded because they either reported they had studied sound symbolism or had participated in a Pokémon-related experiment before. Out of the

5 The other kind of cumulativity is ganging-up cumulativity, in which two different effects add up (Breiss 2020; Jäger and Rosenbach 2006; Kawahara 2020a). A study by D’Onofrio (2014) exemplifies a clear case of ganging-up cumulativity in sound symbolism, in which vowel backness, consonant place of articulation, and consonant voicing all contribute to the strength of the image of roundness (Kawahara 2020a). See Ahlner and Zlatev 2010; Kawahara 2020c; Kawahara and Breiss 2021 for other potential cases of ganging-up cumulativity in sound symbolism. 
remaining participants, fourteen of them were male speakers. Thirty-seven of them were younger than 30 .

\subsection{Results and discussion}

Figure 3 shows the results of the first block-the judged height values. Again, we observe a clear increase in the judged height values as the number of voiced obstruents in the names increases (grand averages: $0=-0.58 ; 1=0.03$; $2=0.54)$. The differences between the two adjacent levels were statistically significant (0 vs. 1: $\beta=-10.9$, s.e. $=2.06, t=-5.27, p<.001 ; 1$ vs. 2 : $\beta=10.5$, s.e. $=2.06, t=5.08, p<.001)$. This result is as predicted by the Frequency Code Hypothesis-voiced obstruents, with their low frequency energy, should be judged to be large (Ohala 1983, 1994). The result is also compatible with the previous judgment experiment on sound symbolism, in which Japanese speakers, as well as English and Chinese speakers, judged nonce words with voiced obstruents to be larger than those without (Shinohara and Kawahara 2016). The result also accords well with other previous studies on sound symbolism such as Bruckert et al. (2006) and Collins (2000), who found that women associate a low-pitched voice with larger body size. The pattern in Figure 3, moreover,

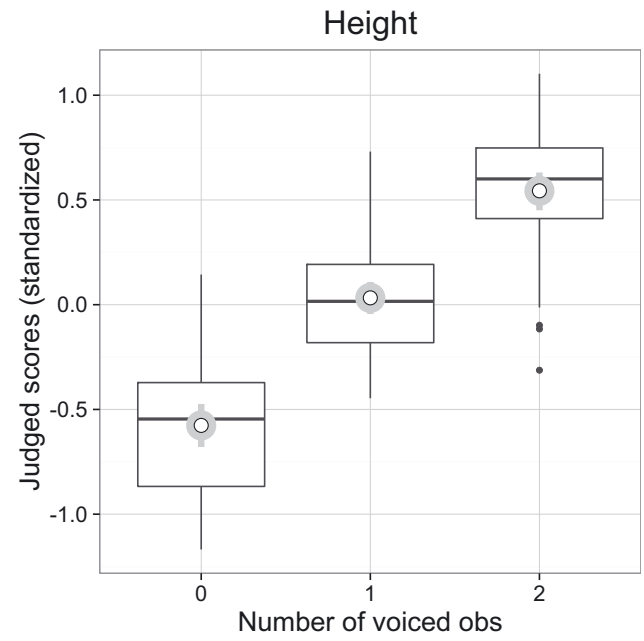

Figure 3: The boxplot representation of the average judged height values for each condition. 
instantiates a clear case of counting cumulativity, in which the judged weight values show a steady increase as a function of the number of voiced obstruents in the stimuli.

Figure 4 represents the distribution of "evolved (=post evolution)" response ratios, which increase as the number of voiced obstruents in the names increases (grand average "post-evolution" response ratios: $0=0.27 ; 1=0.51 ; 2=0.64$ ). The differences between the two adjacent levels were both significant ( 0 vs. 1: $\beta=-1.1$, s.e. $=0.19, z=-5.77, p<.001 ; 1$ vs. $2: \beta=0.57$, s.e. $=0.19, z=3.09, p<.01)$. This result replicates the findings of the other experiments on Pokémon names, in which Japanese participants generally associated names that have voiced obstruents with evolved characters (Kawahara et al. 2018a; Kawahara and Kumagai 2019a; Kawahara, 2020c; Kumagai and Kawahara 2019). Godoy et al. (2020) show a similar effect of voiced obstruents on judged evolution status with Brazilian Portuguese speakers.

While the effects of voiced obstruents on judged evolution levels were generally cumulative, the inspection of the boxplot in Figure 4 shows that there were some participants who judged names with one voiced obstruents to be suited for post-evolution characters $100 \%$ of the time. For some Japanese speakers, then, one voiced obstruent suffices to express evolution; i.e. there is a ceiling effect. The comparison of the grand averages also shows that this effect of voiced obstruents is

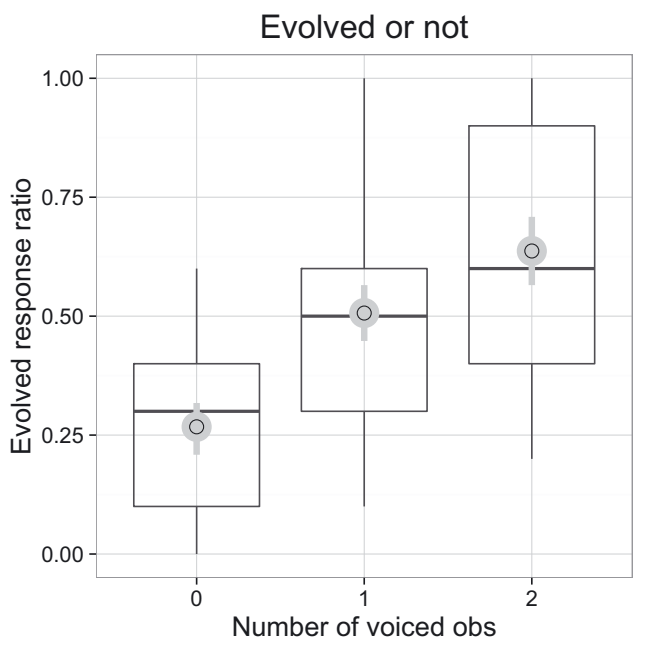

Figure 4: The boxplot representation of the "evolved (=post-evolution)" response ratios. 
sub-linear in the probability domain; i.e. the slope from 0 to 1 is steeper than the slope between 1 and $2^{6}$

\section{General discussion}

The current experiments have shown that Japanese speakers sound symbolically associate voiced obstruents with four semantic dimensions-weight, height, evolution levels and attack values-in the Pokémon universe. These results support the productivity of the sound symbolic patterns that are found in the existing Pokémon names; moreover, the current results are also generally compatible with the findings of other studies on sound symbolism (e.g. Sell et al. 2010 on the relationship between low-pitched voice and strength). While the previous Pokémon-naming experiments (Kawahara et al. 2018a; Kawahara and Kumagai 2019a; Kumagai and Kawahara 2019) have demonstrated the productivity of sound symbolic connections between voiced obstruents and height/evolution levels, the current experiments were the first to experimentally confirm the productivity of the sound symbolic connections between voiced obstruents and weight/strength.

Generally speaking, the results show that one class of sounds-voiced obstruents - can be associated with multiple semantic dimensions. This result in and of itself may not be too surprising, given what Jakobson (1978), one classic writing about sound symbolism in modern linguistics, already stated:

[o]wing to neuropsychological laws of synaesthesia, phonetic oppositions can themselves evoke relations with musical, chromatic, olfactory, tactile, etc. sensations. For example, the opposition between acute and grave phonemes has the capacity to suggest an image of bright and dark, of pointed and rounded, of thin and thick, of light and heavy, etc (p.113)

See also Dingemanse et al. (2015), Hamano (1998), Lindauer (1990), Perlman and Lupyan (2018), Saji et al. (2019), Sidhu and Pexman (2018) and others for multidimenstionality of sound symbolism. In a recent study, Winter et al. (2019) conclude that "the same speech sound is reliably associated with various perceptual, affective, and social meanings. This demonstrates what we call the 'pluripotentiality' of iconicity, that is, the same speech sound is able to trigger a web of interrelated mental associations across different dimensions." The current study further strengthens this conclusion by showing that voiced obstruents in Japanese can symbolically represent various semantic meanings.

6 This sort of sub-linearity in the probability domain does not present an analytical problem for Maximum Entropy Model. See Breiss (2020), Breiss and Albright (2020) and Kawahara (2020a) for related discussion. 
While the main purpose of the paper was to explore which semantic dimensions voiced obstruents can symbolically represent in Japanese, we would like to offer an-admittedly speculative-hypothesis regarding how these multi-dimensional sound symbolic patterns come about. The Frequency Code Hypothesis (Ohala 1983, 1994), one of the most widely discussed theories of sound symbolism, predicts that there is a relationship between size and frequency. Since voiced obstruents involve low frequency energy (Chodroff and Wilson 2014; Kingston and Diehl 1994, 1995; Stevens and Blumstein 1981), they should be associated with large images. Since everything else being equal, large things/animals are heavy (assuming equal density), by extension voiced obstruents can be associated with heavy images (Figure 5). Likewise, large animals are generally expected to be stronger, and similarly, it is not too surprising that the notion of evolution is associated with larger size.

Future studies should seek independent evidence of this two-stage model. We believe that this model is worth further exploration, since as pointed out by Sidhu and Pexman (2018), size (together with shape) is one of the two sound symbolic patterns that is most widely attested in natural languages (see also Berlin 2006; Jespersen 1922; Newman 1933; Nuckolls 1999; Ohala 1983, 1994; Sapir 1929; Shinohara and Kawahara 2009; Taylor and Taylor 1962; Thompson and Estes 2011; Ultan 1978 among many others). Size may be a "privileged" semantic dimension among those meanings that are signaled by sound symbolism, because it is directly inferable from differences in acoustic frequency in the sound. ${ }^{7}$ As stated above, this hypothesis is still speculative, but we believe that it is worth further exploration, given that pluripotentiality of sound

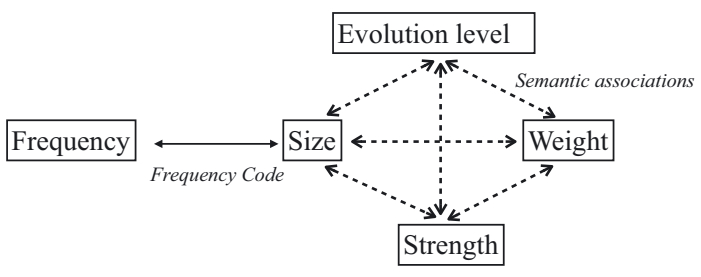

Figure 5: Sound symbolic associations via the Frequency Code and possible semantic extensions. A solid line represents the sound symbolic relationship established via the Frequency Code. Dotted lines represent semantic associations.

\footnotetext{
7 There may be an acquisition stage in which children are sensitive to size-related sound symbolism, but not to other sound symbolic patterns, say, those concerning weight or evolution levels, as the latter relationships need to be mediated by an extra mechanism (e.g. further semantic associations). See Peña et al. (2011) who present evidence that 4-month old infants are demonstrably sensitive to size-related sound symbolism.
} 
symbolism may be one important general feature of sound symbolic patterns in natural languages (Winter et al. 2019).

An anonymous reviewer suggested one type of follow-up experiment that can be used to examine the primacy of a semantic dimension (i.e. size in Figure 5). We could present a pair of two Pokémon characters, in which, for example, one character is larger but lighter, weaker, and less evolved than the other. ${ }^{8}$ If names with voiced obstruents were to be chosen for the larger character, despite it being lighter, weaker, and less evolved, it may show that size, as predicted by the Frequency Code, is the primary semantic dimension associated with voiced obstruents in Japanese. We find this line of research very interesting and promising, but leave this task for future exploration.

A mechanism like the one illustrated in Figure 5 may be at work for other sound symbolic patterns. For example, a distinction between obstruents and sonorants is associated with a visual distinction between angular and round shapes (Köhler 1929 et seq.). Later studies show that the same phonological distinction can also signal personality differences; i.e. "unfriendly and inaccessible type" vs. "friendly and accessible type" (Kawahara et al. 2015; Lindauer 1990; Shinohara and Kawahara 2013; Sidhu et al. 2019), as well as difference in gender (Cassidy et al. 1999; Shinohara and Kawahara 2013; Slater and Feinman 1985). The sound symbolic associations between obstruency and visual shapes may have an acoustic basis-acoustic shapes of obstruents look literally angular on waveforms (Jurafsky 2014; Kawahara and Shinohara 2012; Nobile 2015). On the other hand, the idea that obstruents are "unfriendly" or "male" has no apparent phonetic basis, although it may be not be too surprising if angular shapes can be semantically associated with unfriendliness and maleness. Thus, obstruency can be directly associated with angularity because of acoustic characteristics, and unfriendliness and maleness may be only indirectly associated with obstruency, via semantic associations.

Another case that instantiates pluripotentiality of sound symbolism is that of labial consonants. In Japanese, labial consonants are associated with the notion of babies, appearing very often in diapers' names both in existing words and new words that were created in an elicitation experiment (Kumagai and Kawahara 2020). This association is likely to arise from the fact that labial consonants are those consonants that appear very early in babies' speech and babbling (Jakobson 1941; MacNeilage et al. 1997). A later study has shown, moreover, that labial consonants, in particular [p] and [m], are judged to be “cute" (Kumagai 2019), and are very often used in the names of PreCure fighters (Kawahara 2019) as well as in

8 In the existing Pokémon world, height and size are generally correlated with one another, but such correlation is not perfect. For example, ootachi (1.8 m; $32.5 \mathrm{~kg}$; Pokédex number 162) is larger but lighter than yoogirasu (0.6 m; $72 \mathrm{~kg}$; Pokédex number 246). 
the fairy type Pokémon names (Hosokawa et al. 2018; Kawahara and Kumagai, 2019b; Uno et al. 2020), i.e., characters who are generally "cute.” It is likely that there is nothing inherently "cute" about the articulation or acoustics of labial sounds-labial consonants may be symbolically associated with babies directly, because that is how they acquire speech; this symbolic association can be extended to other "cute" characters, by virtue of the fact that babies are generally perceived to be cute.

In summary, exploring these cases may allow us to address general mechanisms with which certain sets of sounds acquire their sound symbolic meanings. The first step may be via iconic mapping between the phonetic properties of the sounds and their meanings, and these meanings can be further extended via semantic associations. Explicating these mechanisms may shed light on the question of why we observe pluripotentiality of sound symbolism in the first place, and it also opens up interesting research questions. For instance, it would not be too surprising if iconicity-based sound meaning relationships hold across languages, because we use the same articulators in the same physical world. However, semantic associations may not be universal-e.g. it may not be the case that all cultures associate maleness with angularity. The tentative proposal advanced here thus makes specific predictions regarding universality and languagespecificity of sound symbolism. We iterate that the hypothesis presented in Figure 5 is still speculative, which arose from the post-hoc inspection of the current results, but we nevertheless believe that it is worth further exploration.

In addition to confirming the pluripotentiality of sound symbolic values of voiced obstruents in Japanese, another general finding of the current experiments is that the effects of voiced obstruents are cumulative-for each of the semantic dimensions tested, two voiced obstruents systematically evoked stronger images than one voiced obstruents, instantiating what is known as counting cumulativity (Jäger and Rosenbach 2006). This result is compatible with previous findings (Hamano 2013; Kawahara 2020a; Martin 1962; Thompson and Estes 2011), and may support the view that linguistic knowledge, including sound symbolic knowledge, should be modeled with a mechanism that naturally predicts this sort of cumulativity effects, e.g. Maximum Entropy Harmonic Grammar (Kawahara 2020a, 2020c).

Acknowledgments: We are grateful to four anonymous JJL reviewers as well as Donna Erickson for their comments on previous versions of this paper. We would also like to thank the editor for his editorial guidance, as well as to all the participants of the experiments for sharing their knowledge about Japanese. This project is supported by the JSPS grants (\#17K13448, \#18H03579, and \#19K13164). All remaining errors are ours. 


\section{References}

Ahlner, Felix \& Jordan Zlatev. 2010. Cross-modal iconicity: A cognitive semiotic approach to sound symbolism. Sign Sytems Studies 38(1/4). 298-348.

Alderete, John \& Alexei Kochetov. 2017. Integrating sound symbolism with core grammar: The case of expressive palatalization. Language 93. 731-766.

Barr, Dale J. 2013. Random effects structure for testing interactions in linear mixed-effects models. Frontiers in Psychology 4. 328.

Barr, Dale J., Roger Levy, Christoph Scheepers \& Harry J. Tily. 2013. Random effects structure for confirmatory hypothesis testing: Keep it maximal. Journal of Memory and Language 68. 255-278.

Bates, Douglas, Martin Maechler, Ben Bolker, Steven Walker, Rune Haubo Bojesen Christensen, Henrik Singmann, Bin Dai, Fabian Scheipl, Gabor Grothendieck \& Peter Green. 2017. Ime4. r-package.

Berlin, Brent. 2006. The first congress of ethonozoological nomenclature. Journal of Royal Anthropological Institution 12. 23-44.

Blasi, Damián, Søren Wichman, Harald Hammarström, Peter F. Stadler \& Morten H. Christianson. 2016. Sound-meaning association biases evidenced across thousands of languages. Proceedings of the National Academy of Sciences 113(39). 10818-10823.

Breiss, Canaan. 2020. Constraint cumulativity in phonotactics: Evidence from artificial grammar learning studies. Ms. UCLA. https://doi.org/10.1353/lan.0.0243.

Breiss, Canaan \& Adam Albright. 2020. Cumulative markedness effects and (non-)linearity in phonotactics. Ms. UCLA and MIT. https://doi.org/10.1353/lan.0.0243.

Browman, Catherine. 1978. Tip of the tongue and slip of the ear: Implications for language processing. UCLA Working Papers in Phonetics 42.

Bruckert, Laetitia, Liénard Jean-Sylvain, André Lacroix, Michel Kreutzer \& Gérard Leboucher. 2006. Women use voice parameters to assess men's characteristics. Proceedings of the Royal Society of London B Biological Sciences 273. 83-89.

Cassidy, Kimberly Wright, Michael H. Kelly \& Lee'at J. Sharoni. 1999. Inferring gender from name phonology. Journal of Experimental Psychology: General 128. 362-381.

Chodroff, Eleanor \& Colin Wilson. 2014. Burst spectrum as a cue for the stop voicing contrast in American English. Journal of the Acoustical Society of America 136(5). 2762-2772.

Collins, Sarah A. 2000. Men's voices and women's choices. Animal Behaviour 60(6). 773-780.

Dingemanse, Mark, Damián E. Blasi, Lupyan Gary, Morten H. Christiansen \& Padraic Monaghan. 2015. Arbitrariness, iconicity and systematicity in language. Trends in Cognitive Sciences 19(10). 603-615.

D’Onofrio, Annette. 2014. Phonetic detail and dimensionality in sound-shape correspondences: Refining the bouba-kiki paradigm. Language and Speech 57(3). 367-393.

Godoy, Mahayana C., Neemias Silva de Souza Filho, Juliana G. Marques de Souza, Hális Alves \& Shigeto Kawahara. 2020. Gotta name'em all: An experimental study on the sound symbolism of Pokémon names in Brazilian Portuguese. Journal of Psycholinguistic Research 49. 717-740.

Goldwater, Sharon \& Mark Johnson. 2003. Learning OT constraint rankings using a maximum entropy model. In Proceedings of the Workshop on Variation within Optimality Theory, 111-120. 
Gussenhoven, Carlos. 2004. The phonology of tone and intonation. Cambridge: Cambridge University Press.

Gussenhoven, Carlos. 2016. Foundations of intonational meaning: Anatomical and physiological factors. Topics in Cognitive Science 8. 425-434.

Hamano, Shoko. 1998. The sound-symbolic system of Japanese. Stanford: CSLI Publications.

Hamano, Shoko. 2013. Hoogen-ni okeru giongo-gitaigo-no taikeiteki-kenkyuu-no igi. In Kazuko Shinohara \& Ryoko Uno (eds.), Chikazuku oto-to imi: Onomatope kenkyuu-no shatei, 133-147. Tokyo: Hitsuzi Syobo.

Hawkins, John \& Anne Cutler. 1988. Psycholinguistic factors in morphological asymmetry. In J. A. Hawkins (ed.), Explaining language universals, 280-317. Oxford: Basil Blackwell.

Hayes, Bruce \& Colin Wilson. 2008. A maximum entropy model of phonotactics and phonotactic learning. Linguistic Inquiry 39. 379-440.

Hayes, Bruce, Colin Wilson \& Anne Shisko. 2012. Maxent grammars for the metrics of Shakespeare and Milton. Language 88(4). 691-731.

Hockett, Charles. 1959. Animal "languages" and human language. Human Biology 31. 32-39.

Hosokawa, Yuta, Naho Atsumi, Ryoko Uno \& Kazuko Shinohara. 2018. Evil or not? Sound symbolism in Pokémon and Disney character names. In Talk presented at the 1st international conference on Pokémonastics.

Jaeger, Florian T. 2008. Categorical data analysis: Away from ANOVAs (transformation or not) and towards logit mixed models. Journal of Memory and Language 59. 434-446.

Jäger, Gerhad \& Anette Rosenbach. 2006. The winner takes it all-almost: Cumulativity in grammatical variation. Linguistics 44(5). 937-971.

Jakobson, Roman. 1941. Child language, aphasia and phonological universals. The Hague: Mouton. Translated into English by A. Keiler, 1968.

Jakobson, Roman. 1978. Six lectures on sound and meaning. Cambridge: MIT Press.

Jespersen, Otto. 1922. Symbolic value of the vowel $i$. In Linguistica: Selected papers in English, French and German, vol. 1, 283-303. Copenhagen: Levin and Munksgaard.

Johansson, Niklas \& Jordan Zlatev. 2013. Motivations for sound symbolism in spatial deixis: A typological study of 101 languages. The Public Journal of Semiotics 5(1). 1-20.

Jurafsky, Dan. 2014. The language of food: A linguist reads the menu. New York: W. W. Norton \& Company.

Kawahara, Shigeto. 2017. Introducing Phonetics through Sound Symbolism. Tokyo: Hitsuzi Syobo.

Kawahara, Shigeto. 2019. What's in a precure name? ICU Working Papers in Linguistics 7: Festschrift for Professor Tomoyuki Yoshida on his 60th birthday, 15-22.

Kawahara, Shigeto. 2020a. Cumulative effects in sound symbolism. Ms. Keio University.

Kawahara, Shigeto. 2020b. Sound symbolism and theoretical phonology. Language and Linguistic Compass 14(8). e12372.

Kawahara, Shigeto. 2020c. A wug-shaped curve in sound symbolism: The case of Japanese Pokémon names. Phonology 37(3). https://doi.org/10.1017/s0952675720000202.

Kawahara, Shigeto \& Canaan Breiss. 2021. Exploring the nature of cumulativity in sound symbolism: Experimental studies of Pokémonastics with English speakers. Ms. Keio University and UCLA.

Kawahara, Shigeto, Miwa Isobe, Yukino Kobayashi, Tomoko Monou \& Reiko Okabe. 2018a. Acquisition of sound symbolic values of vowels and voiced obstruents by Japanese children: Using a Pokémonastics paradigm. Journal of the Phonetic Society of Japan 22(2). 122-130.

Kawahara, Shigeto, Hironori Katsuda \& Gakuji Kumagai. 2019. Accounting for the stochastic nature of sound symbolism using Maximum Entropy model. Open Linguistics 5. 109-120. 
Kawahara, Shigeto \& Gakuji Kumagai. 2019a. Expressing evolution in Pokémon names: Experimental explorations. Journal of Japanese Linguistics 35(1). 3-38.

Kawahara, Shigeto \& Gakuji Kumagai. 2019b. Inferring Pokémon types using sound symbolism: The effects of voicing and labiality. Journal of the Phonetic Society of Japan 23(2). 111-116.

Kawahara, Shigeto, Atsushi Noto \& Gakuji Kumagai. 2018b. Sound symbolic patterns in Pokémon names. Phonetica 75(3). 219-244.

Kawahara, Shigeto \& Kazuko Shinohara. 2012. A tripartite trans-modal relationship between sounds, shapes and emotions: A case of abrupt modulation. Proceedings of CogSci. 569-574.

Kawahara, Shigeto, Kazuko Shinohara \& Joseph Grady. 2015. Iconic inferences about personality: From sounds and shapes. In Masako Hiraga, William Herlofsky, Kazuko Shinohara \& Kimi Akita (eds.), Iconicity: East meets west, 57-69. Amsterdam: John Benjamins.

Kawahara, Shigeto, Kazuko Shinohara \& Yumi Uchimoto. 2008. A positional effect in sound symbolism: An experimental study. In Proceedings of the Japan Cognitive Linguistics Association 8, 417-427. Tokyo: JCLA.

Kawahara, Shigeto, Michinori Suzuki \& Gakuji Kumagai. 2020. Sound symbolic patterns in Pokémon move names in Japanese. ICU Working Papers in Linguistics 10. Festschrift for Prof. Junko Hibiya in the occasion of her retirement from ICU 17-30.

Kingston, John \& Randy Diehl. 1994. Phonetic knowledge. Language 70. 419-454.

Kingston, John \& Randy Diehl. 1995. Intermediate properties in the perception of distinctive feature values. In Bruce Connell \& Amalia Arvaniti (eds.), Papers in laboratory phonology IV: Phonology and phonetic evidence, 7-27. Cambridge: Cambridge University Press.

Köhler, Wolgang. 1929. Gestalt psychology. New York: Liveright.

Kubozono, Haruo. 1999. Nihongo-no onsei: Gendai gengogaku nyuumon 2 [Japanese phonetics: An introduction to modern linguisitcs 2]. Tokyo: Iwanami Shoten.

Kumagai, Gakuji. 2019. A sound-symbolic alternation to express cuteness and the orthographic Lyman's Law in Japanese. Journal of Japanese Linguistics 35(1). 39-74.

Kumagai, Gakuji \& Shigeto Kawahara. 2019. Effects of vowels and voiced obstruents on Pokémon names: Experimental and theoretical approaches [in Japanese]. Journal of the Linguistic Society of Japan 155. 65-99.

Kumagai, Gakuji \& Shigeto Kawahara. 2020. How abstract is sound symbolism? Labiality and diaper names in Japanese [in Japanese]. Journal of the Linguistic Society of Japan 157. 149-161.

Kuznetsova, Alexandra, Per Bruun Brockhoff \& Rune Haubo Bojesen Christensen. 2017. Imertest, r-package.

Legendre, Géraldine, Yoshiro Miyata \& Smolensky Paul. 1990. Harmonic grammar - A formal multi-level connectionist theory of linguistic well-formedness: Theoretical foundations. In Proceedings of the Twelfth Annual Conference of the Cognitive Science Society, 388-395. Mahwah, NJ: Lawrence Erlbaum Associates.

Lindauer, S. Martin. 1990. The meanings of the physiognomic stimuli taketa and maluma. Bulletin of the Psychonomic Society 28(1). 47-50.

Lockwood, Gwilym \& Mark Dingemanse. 2015. Iconicity in the lab: A review of behavioral, developmental, and neuroimaging research into sound-symbolism. Frontiers in Psychology. https://doi.org/10.3389/fpsyg.2015.01246.

MacNeilage, Peter F., Barbara L. Davis \& Christine L. Matyear. 1997. Babbling and first words: Phonetic similarities and differences. Speech Communication 22(2-3). 269-277.

Martin, Samuel. 1962. Phonetic symbolism in Korean. In N. Poppe (ed.), American studies in Uralic and Altaic linguistics. Indiana University Press. 
McCarthy, John J. 1983. Phonological features and morphological structure. In J. Richardson, M. Marks \& A. Chukerman (eds.), Proceedings from the parasesion on the interplay of phonology, morphology and syntax, 135-161. Chicago: CLS.

McPherson, Laura \& Bruce Hayes. 2016. Relating application frequency to morphological structure: The case of Tommo So vowel harmony. Phonology 33. 125-167.

Newman, Stanley. 1933. Further experiments on phonetic symbolism. American Journal of Psychology 45. 53-75.

Nobile, Luca. 2015. Phonemes as images: An experimental inquiry into shape-sound symbolism applied to the distinctive features of French. In Masako Hiraga, William Herlofsky, Kazuko Shinohara \& Kimi Akita (eds.), Iconicity: East meets west, 71-91. Amsterdam: John Benjamins.

Nooteboom, Sieb. 1981. Lexical retrieval from fragments of spoken words: Beginnings vs. endings. Journal of Phonetics 9. 407-424.

Nuckolls, Janis B. 1999. The case for sound symbolism. Annual Review of Anthropology 28. 225-252.

Ohala, John. 1983. Cross-language use of pitch: An ethnological view. Phonetica 40. 1-18.

Ohala, John. 1994. The frequency code underlies the sound symbolic use of voice pitch. In Leane Hinton, Johanna Nichols \& John Ohala (eds.), Sound symbolism, 325-347. Cambridge: Cambridge University Press.

Pater, Joe. 2009. Weighted constraints in generative linguistics. Cognitive Science 33. 999-1035.

Peña, Marcela, Jacques Mehler \& Marina Nespor. 2011. The role of audiovisual processing in early conceptual development. Psychological Science 22(11). 1419-1421.

Perlman, Marcus \& Gary Lupyan. 2018. People can create iconic vocalizations to communicate various meanings to naïve listeners. Scientific Reports 26-34.

Pitcher, Benjamin J., Alex Mesoudi \& Alan G. McElligott. 2013. Sex-based sound symbolism in English-language first names. PLoS One 8(6). e64825.

Prince, Alan \& Paul Smolensky. 1993/2004. Optimality Theory: Constraint interaction in generative grammar. Malden and Oxford: Blackwell.

R Development Core Team. 1993. R: A language and environment for statistical computing. Austria: R Foundation for Statistical Computing Vienna.

Saji, Noburo, Kimi Akita, Katerina Kantartzis, Sotaro Kita \& Mutsumi Imai. 2019. Crosslinguistically shared and language-specific sound symbolism in novel words elicited by locomotion videos in Japanese and English. PLoS One 14(7). e0218707.

Sapir, Edward. 1929. A study in phonetic symbolism. Journal of Experimental Psychology 12. 225-239.

Saussure, Ferdinand de. 1916. Cours de linguistique générale. Paris: Payot.

Sell, Aaron, Gregory A. Bryant, Leda Cosmides, John Tooby, Daniel Sznycer, Christopher Von Rueden, Andre Krauss \& Michael Gurven. 2010. Adaptations in humans for assessing physical strength from the voice. Proceedings of the Royal Society of London $B$ Biological Sciences. https://doi.org/10.1098/rspb.2010.0769.

Shih, Stephanie S, Jordan Ackerman, Noah Hermalin, Sharon Inkelas, Hayeun Jang, Jessica Johnson, Darya Kavitskaya, Shigeto Kawahara, Miran Oh, Rebecca L Starr \& Alan Yu. 2019. Cross-linguistic and language-specific sound symbolism: Pokémonastics. Ms. University of Southern California, University of California, Merced, University of California. Berkeley: Keio University, National University of Singapore and University of Chicago.

Shih, Stephanie S., Jordan Ackerman, Noah Hermalin, Sharon Inkelas \& Darya Kavitskaya. 2018. Pokémonikers: A study of sound symbolism and Pokémon names. Proceedings of LSA 2018. 
Shinohara, Kazuko \& Shigeto Kawahara. 2009. A cross-linguistic study of sound symbolism: Images evoked by voicing. In Proceedings of the 26th Annual Meeting of Japanese Cognitive Science Society.

Shinohara, Kazuko \& Shigeto Kawahara. 2013. The sound symbolic nature of Japanese maid names. In Proceedings of the 13th Annual Meeting of the Japanese Cognitive Linguistics Association 13, 183-193.

Shinohara, Kazuko \& Shigeto Kawahara. 2016. A cross-linguistic study of sound symbolism: The images of size. In Proceedings of the Thirty Sixth Annual Meeting of the Berkeley Linguistics Society, 396-410. Berkeley: Berkeley Linguistics Society.

Sidhu, David \& Penny M. Pexman. 2018. Five mechanisms of sound symbolic association. Psychonomic Bulletin \& Review 25(5). 1619-1643.

Sidhu, David \& Penny M. Pexman. 2019. The sound symbolism of names. Current Directions in Psychological Science 28(4). 398-402.

Sidhu, David M., Kristen Deschamps, Joshua S. Bourdage \& Penny M. Pexman. 2019. Does the name say it all? Investigating phoneme-personality sound symbolism in first names. Journal of Experimental Psychology: General 148(9). 1595-1614.

Slater, Anne Saxon \& Saul Feinman. 1985. Gender and the phonology of North American first names. Sex Roles 13. 429-440.

Spence, Charles. 2011. Crossmodal correspondences: A tutorial review. Attention. Perception \& Psychophysics 73(4). 971-995.

Stevens, Kenneth \& Sheila Blumstein. 1981. The search for invariant acoustic correlates of phonetic features. In Peter Eimas, D. Joanne \& Miller (eds.), Perspectives on the study of speech, 1-38. New Jersey: Earlbaum.

Suzuki, Takao. 1962. Oninkookan to igibunka no kankei ni tsuite-iwayuru seidakuon tairitsu-o chuushin toshite. Gengo Kenkyu [Journal of the Linguistic Society of Japan] 42. 23-30.

Taylor, I. K. \& M. M. Taylor. 1962. Phonetic symbolism in four unrelated languages. Canadian Journal of Experimental Psychology 16. 344-356.

Thompson, Patrick D. \& Zachary Estes. 2011. Sound symbolic naming of novel objects is a graded function. Quarterly Journal of Experimental Psychology 64(12). 2392-2404.

Ultan, Russell. 1978. Size-sound symbolism. In Joseph Greenberg (ed.), Universals of human language II: Phonology, 525-568. Stanford: Stanford University Press.

Uno, Ryoko, Kazuko Shinohara, Yuta Hosokawa, Naho Ataumi, Gakuji Kumagai \& Shigeto Kawahara. 2020. What's in a villain's name? Sound symbolic values of voiced obstruents and bilabial consonants. Annual Review of Cognitive Linguistics 18(2). 428-457.

Westbury, Chris. 2005. Implicit sound symbolism in lexical access: Evidence from an interference task. Brain and Language 93. 10-19.

Wichmann, Søren, Eric W. Holman \& Cecil H. Brown. 2010. Sound symbolism in basic vocabulary. Entropy 12(4). 844-858.

Winter, Bodo, Paula Pérez-Sobrino \& Lucien Brown. 2019. The sound of soft alcohol: Crossmodal associations between interjections and liquor. PLoS One 14(8). e0220449. 